Meta

Journal des traducteurs

Translators' Journal

\title{
Discours, lexique, dictionnaire
}

\section{Louis Guilbert}

Volume 18, numéro 1-2, mars 1973

Actes du deuxième colloque international de linguistique et de traduction. Montréal, 4-7 octobre 1972

URI : https://id.erudit.org/iderudit/002983ar

DOI : https://doi.org/10.7202/002983ar

Aller au sommaire du numéro

Éditeur(s)

Les Presses de l'Université de Montréal

ISSN

0026-0452 (imprimé)

1492-1421 (numérique)

Découvrir la revue

Citer cet article

Guilbert, L. (1973). Discours, lexique, dictionnaire. Meta, 18(1-2), 201-224.

https://doi.org/10.7202/002983ar d'utilisation que vous pouvez consulter en ligne.

https://apropos.erudit.org/fr/usagers/politique-dutilisation/ 


\section{Discours, \\ lexique, dictionnaire}

Lorsque nous formulons cette trilogie, notre intention n'est pas de traiter de tous les problèmes que soulève chacun de ces termes; c'est l'objet de toute la linguistique : mais seulement d'aborder un aspect de la relation que l'on peut établir entre les réalités linguistiques qu'ils désignent. Notre réflexion prendra comme paramètre l'unité de fonctionnement du langage, si mal définie malgré son omniprésence - le mot. Nous n'avons pas non plus l'intention de tenter à notre tour une théorie du mot. Notre propos est d'examiner certains aspects du rapport entre la phrase et les éléments lexicaux et d'en tirer des conclusions du point de vue de la description du lexique, de la fabrication du dictionnaire.

\section{LE MOT, SA PROBLÉMATIQUE}

1.1. On prendra comme définition opérationnelle du mot celle de Meillet, \& une tranche sonore douée d'une fonction syntaxique et pourvue d'un sens $\$$. Le premier élément de la définition implique une concordance absolue entre l'unité graphique de la langue écrite et l'unité sonore de la langue parlée. Mais chacun peut constater que la réalité du langage est toute différente. Par exemple, la tranche sonore du langage parlé correspondant au mot écrit est inséparable du déterminant portant les marques du genre et du nombre, surtout dans les mots à initiale vocalique les yeux [lezjø], l'eau [lo], sauf dans une situation métalinguistique (les mots yeux ou eau) ; les unités sonores gendarme [3ãdarm], chauffe-eau [šofo], point de vue [pwẽdvy] sont identiques quant à la cohésion des phonèmes constituants; alors que, dans la graphie, l'une est une unité lexicale soudée, l'autre comporte deux éléments lexicaux reliés par un trait d'union et la troisième est formée de trois éléments non reliés.

En ce qui concerne le sens, la séquence lexicale sécurité sociale ne s'analyse pas par l'addition des traits sémiques afférents à la tranche sonore sécurité et à la tranche sonore sociale, mais par la référence unique à la garantie offerte par cette institution. Quant à la fonction syntaxique, il semble évident que toute unité lexicale doive s'inscrire dans une classe syntaxique en tant qu'élément de phrase. Cependant, certains éléments sonores appelés communément mots apparaissent difficilement classables parmi les fonctions syntaxiques du mot constituant de la phrase, les interjections et les exclamations, alors qu'ils répondent à la définition du signe linguistique - comme union d'un signifiant unique d'un 
signifié. Telles sont quelques-unes des nombreuses questions soulevées par cette forme linguistique du mot.

1.2. Les difficultés rencontrées dans la délimitation et la définition du mot relèvent pour l'essentiel du problème fondamental de la relation entre la syntaxe et le lexique : ou bien les mots sont avant tout des unités de sens, en tant que signes de référence à une réalité matérielle, conceptuelle ou psychologique; ils se combinent selon des rapports logiques dont la grammaire fournit le schéma syntaxique sous forme de règles de formation de la phrase, afin de permettre une communication codifiée et normalisée entre les individus d'une même communauté linguistique. Ou bien le schéma de la phrase est prioritaire dans le fonctionnement du langage, fournissant à chaque individu parlant le cadre où viennent s'insérer les mots, soit qu'ils s'y juxtaposent en fonction d'une certaine sélection sémantique : [Le ciel est bleu, clair, gris, nuageux], soit qu'ils réagissent sur la structuration de la phrase par la sélection sémantique qu'ils impliquent. La définition du lexique et la méthode de description se trouveront profondément modifiées selon l'option choisie. Dans le premier cas, le lexique n'est qu'un catalogue de signifiants, réceptacles d'un certain nombre de traits de signification, dotés d'une fonction syntaxique déterminée une fois pour toutes : substantif, verbe, adjectif, etc., les traditionnelles catégories du discours. Au contraire, si la phrase est le principe premier du langage, il s'ensuit que la relation syntaxique est le principe de production du langage, prioritaire par rapport au faisceau sémique qu'elle détermine : le mot est d'abord défini par son aptitude à se combiner selon le schéma fondamental de la phrase.

1.3. L'ambiguïté du terme mot provient du fait que, par commodité d'expression, en vertu du poids de l'usage et des analyses linguistiques antérieures, on use de ce lexème pour se référer à des fonctions différentes dans le lexique. Il désigne, en effet, à la fois l'unité constitutive de la chaîne syntaxique de la phrase dans la performance du discours, et la forme signifiante d'un certain contenu de signification de l'expérience humaine, du monde à travers le locuteur d'une communauté linguistique.

Il conviendrait donc d'abord de bien définir ce qu'est l'unité lexicale en tant qu'élément de formation de la phrase, même si l'on doit en définitive recourir au terme mot par paresse. Cette définition ne peut être produite en toute clarté que dans la théorie de la grammaire générative et transformationnelle. Si l'on se borne, en effet, à rendre compte de la combinaison d'éléments lexicaux par la simple cohérence de juxtaposition d'éléments morphologiquement et sémantiquement autonomes, par la globalisation de ces éléments de signification, on passe à côté du véritable processus de création, du principe d'union de ces éléments.

La grammaire générative permet d'expliquer la relation syntaxique entre les éléments lexicaux à partir d'une phrase de base qui les contient et le processus d'intégration de ces éléments par la transformation de la séquence de phrase en combinaison lexicale. Soient les deux séquences : 1) La foule applaudit; cela (ça) stimule l'haltérophile; 2) Les applaudissements de la foule stimulent l'haltérophile. 
À ne considérer que le résultat de la transformation dans la séquence 2, on constate que la formation lexicale applaudissements représente sous une forme nominale l'action exprimée par la forme du verbe dans la séquence 1, qu'au morphème-désinence portant la marque du temps, du mode, de la personne dans le verbe s'est substitué l'affixe créateur de la catégorie nominale et porteur du trait «action», que le syntagme nominal sujet est devenu syntagme nominal complément du nom par l'entremise de l'opérateur de. On remarquera que la transformation nominale implique, outre la mutation morphologique par l'affixe, la formation de la nouvelle séquence syntagmatique $\mathrm{SN}+\mathrm{de}+\mathrm{SN}$ et que la création du nom est intégrée à un processus syntagmatique. La transformation affecte le trait essentiel de la catégorie grammaticale en tant que telle. L'opération s'accomplit au niveau de la classe syntaxique : c'est pourquoi on parle parfois de morphosyntaxe pour désigner ce processus de création. Dans cette perspective, les traditionnelles catégories du discours ne sont plus toutes mises sous le même plan. On distingue les classes syntaxiques dynamiques (verbe, substantif, adjectif) qui créent les transformations lexicales dans des processus de verbalisation de substantivation et d'adjectivation, tandis que les éléments lexicaux, dits ordinairement «mots grammaticaux» (déterminants, prépositions, conjonctions) sont des opérateurs syntaxiques dans la combinaison des éléments de la phrase. Ainsi le lexème générateur cesse d'être un élément lexical figé dans une classe ; il devient un élément actif d'organisation de la phrase et de création permanente dans le discours du locuteur. La créativité de celui-ci ne se caractérise pas seulement par la faculté de combiner les mots d'une manière nouvelle à partir du schéma fondamental de la phrase et de ses diverses transformations, mais aussi par la faculté de produire de nouvelles formes lexicales.

\section{LE MORPHÈME LEXICAL}

2.1. Le mot, en grammaire générative et transformationnelle, qu'on pourrait appeler morphème lexical, se caractérise donc essentiellement par la classe syntaxique dont il tient ses facultés de combinaison. Il apparaît comme une étape dans un processus permanent de transformation en vertu duquel il peut passer d'une classe dans une autre, selon les nécessités de la combinaison de la phrase - en vertu d'une chaîne de transformations diverses de classe à classe - : verbe $\rightarrow$ substantif, substantif $\rightarrow$ verbe, adjectif $\rightarrow$ verbe, verbe $\rightarrow$ adjectif, substantif $\rightarrow$ adjectif, adjectif $\rightarrow$ substantif, mais aussi à l'intérieur d'une même classe : verbe $\rightarrow$ verbe, substantif $\rightarrow$ substantif, adjectif $\rightarrow$ adjectif.

Mais aucun segment linguistique réalisé en phrase ne saurait se concevoir en dehors de l'union d'un signifiant et d'un signifié. $\mathrm{Il}$ convient donc de définir le contenu sémantique du morphème lexical. Il comporte d'abord les traits spécifiques de la classe syntaxique - verbe : «action», procès + agent du procès/copule - substantif : dénomination et substance ou qualité-adjectif : qualité, appartenance, en vertu desquels opère la combinatoire syntaxique de chacun : $\mathbf{N}+\mathrm{V}-\mathrm{N}+$ Adj excluant Adj $+\mathrm{V}$ sauf sous la forme de l'adjectif du verbe - l'adverbe. Ces relations rejoignent le rapport prédicatif fondamental constitutif de la phrase sous la forme sujet + verbe ou substantif + adjectif - par l'inter- 
médiaire de la copule être. Le niveau sémantique le plus général du morphème lexical apparaît avec évidence lorsqu'intervient la transformation lexicale, appelée parfois conversion, et qui consiste, pour un morphème demeurant morphologiquement identique, dans le changement de classe syntaxique : la substantivation de l'adjectif (l'utile, l'agréable), l'adjectivation du substantif (Il est tarte [fam.]). Ce niveau sémantique qui tient à l'essence de la classe syntaxique appartient à la «structure profonde » du morphème lexical.

Celui-ci se définit aussi par des traits sémantiques qui opèrent une sélection parmi les membres de chaque classe virtuellement possibles dans la constitution de la phrase, et qui déterminent les règles de compatibilité sémantique entre les éléments lexicaux de la phrase réalisée. Soit le verbe brûler : il comporte, en plus des sèmes spécifiques de la classe verbe, les sèmes «procès de combustion 》 « action naturelle » qui oriente la phrase vers une réalisation en tant que procès intransitif de valeur passive [La forêt brâle] $=$ la forêt est brûlée], exclusive de l'agent du procès, c'est-à-dire de la forme transitive. Celle-ci n'intervient que par une transformation qui introduit l'agent dans le procès [faire que $\mathrm{N}$ est brûlé $\rightarrow$ brûler]. L'emploi du verbe brûler avec ses traits sémantiques fondamentaux détermine une certaine structure de phrase : $\mathrm{SN}^{1}+\mathrm{V}$ dans le premier cas, $\mathrm{SN}^{1}+\mathrm{SV}$ $\left[\mathrm{V}+\mathrm{SN}^{2}\right]$ dans le second cas. La sélection sémantique de brûler en tant que morphème lexical s'arrête-t-elle à ce niveau, purement syntactico-sémantique, ou opère-t-elle un choix jusque parmi les différents lexèmes membres de la classe syntaxique $\mathrm{SN}^{1}$ et $\mathrm{SN}^{2}$ en fonction des composants sémiques de chacun d'eux ? L'énumération des matières susceptibles de combustion relève de la description du sème combustion, de la nomenclature de la chimie. Le niveau du morphème lexical impliquera seulement dans le $\mathrm{SN}^{1}$ du verbe brûler intransitif le sème «matière combustible ». De même, la détermination des agents du procès brûler dans la construction transitive, au niveau du lexème, se bornera à l'apposition agent naturel [Le soleil brûle la terre] agent humain [Les Anglais ont brûlé Jeanne $\left.d^{\prime} A r c\right]$. En morpho-syntaxe, les traits sémiques à valeur sélective se ramènent à une structure simplifiée : animé vs non-animé, humain vs non-humain, matière $v s$ non-matière, phénomène naturel $v s$ activité humaine, quantifiable $v s$ non-quantifiable, numérable $v s$ non-numérable, spatialité $v s$ non-spatialité, durée $v s$ nondurée, tous les traits qui rendent compte des structures les plus générales de la phrase. On peut se demander si cette structure syntactico-sémantique qui détermine la phrase réalisée ne subit pas des adaptations particulières chez des locuteurs qui ont à exprimer des significations particulières en raison de l'objet de leur discours spécial. C'est un problème posé, par exemple, par les recherches sur le vocabulaire général d'orientation scientifique.

2.2. Dans quelle mesure le morphème lexical peut-il être défini comme un élément lexical indépendant de la phrase ? N'est-il pas nécessaire de le considérer comme tel lorsqu'un nouvel élément lexical procède de lui ? C'est là un faux problème. Le morphème lexical, en effet, par sa fonction syntagmatique, est un élément de transformation permanente. Celle-ci peut revêtir un double aspect ; ou bien, elle répond à un besoin stylistique de permutation dans la phrase du type : La foule applaudit $\rightarrow$ les applaudissements de la foule, ou bien elle donne lieu à une 
création lexicale, du type : Les terroristes focalisent l'attention du monde $\rightarrow$ la focalisation de l'attention du monde. Peu importe que applaudissements soit un lexème ancien et focalisation un mot créé, un néologisme. Ils procèdent tous deux de la même transformation, de la même règle. Le morphème lexical est, par sa fonction syntagmatique, nécessairement base de création. Dans cette perspective, la néologie est une conséquence régulière de l'activité langagière. Le statut sémantique et social ultérieur du terme créé relève de facteurs qui se situent sur le plan de la communauté linguistique, à la fois de la sémantique, de la psycho-linguistique et de la socio-linguistique.

2.3. En vertu de sa disponibilité pour toutes les transformations lexicales selon des règles définies, le morphème lexical fonctionne comme base de dérivation multidirectionnelle, pourrait-on dire. Il se constitue au cours de l'activité langagière des chaînes de dérivation qui rassemblent dans une même classe paradigmatique des morphèmes lexicaux comportant un même noyau morphologique et sémantique. Cependant, ces chaînes lexicales sont d'essence syntagmatique, car l'élément qui rassemble ces dérivés réside dans certains types de transformations de phases de base qui peuvent se présenter théoriquement dans une certaine successivité. Ces chaînes lexicales sont différentes des chaînes à base étymologique. Celles-ci se structurent seulement à partir d'un noyau sémantique et morphologique originel commun, indépendamment des évolutions sémantiques subies par le mot à travers les siècles. Selon cette structuration, on trouve sous la base bûche, bûcher (n), bûcher (v), bûchage, bûcheur, bûcheron, bûcheronner, bûcheronnage, bûchette. Le seul élément constitué par le faisceau de sèmes attaché à une forme qu'on présente d'ailleurs sous l'aspect morphologique du latin busca, bûche n'étant qu'une étape dans la carrière historique de l'étymon. Selon le fonctionnement synchronique de la langue et la filiation syntagmatique théorique, on dégage trois morphèmes lexicaux bases : 1) bûche $\rightarrow$ bûcher (v) $\rightarrow$ bûcheron $\rightarrow$ bûcher (n) $\rightarrow$ bûchette ; 2) bûcheron bûcheronner bûcheronnage; 3 ) bûcher $(v) \rightarrow$ bûchage $\rightarrow$ bûcheur. On remarquera que, selon cette répartition, chaque terme d'une chaîne est étroitement dépendant du terme générateur par la transformation qui la fait surgir. Il ne peut y avoir d'écart sémantique important dans le dérivé par rapport à la base. Les séries transformationnelles sont donc homogènes sémantiquement puisque selon le principe même de la transformation, le contenu sémantique initial du morphème base doit se retrouver intégralement dans le dérivé, adapté morphologiquement à sa nouvelle fonction syntaxique. Il s'ensuit qu'une communauté morphologique de l'élément de base et même une parenté sémantique générale est insuffisante pour fonder une série lexicale transformationnelle. Celle-ci implique une filiation directe et une récursivité permanente de la transformation. C'est ainsi qu'il faut expliquer, pour une part, les dérivés - frères-siamois du type thêâtraliser, théâtralisation, théâtral, théatralité.

Le principe constitutif de la série lexicale transformationnelle implique des séries différenciées sémantiquement à partir d'un même morphème lexical de base. Diverses combinaisons peuvent alors se produire. Il peut arriver qu'un morphème lexical de base soit constitué en chef de file, en vertu de son autonomie sémantique, malgré l'homonymie morphologique (ex. : bûcher 1, faire des bûches 
et bûcher 2, «travailler dur »), et engendre une série autonome sémantiquement (bûchage, bûcher n'existent que par rapport au contenu sémantique de bûcher 2). Parfois chacun des morphèmes lexicaux, homonymiques morphologiquement, mais différenciés sémantiquement, produit la série complète : l'homonymie existe alors pour toute la chaîne : câble 1) (cordage) $\rightarrow$ câbler - câblage; câble 2) (dépêche) $\rightarrow$ câbler $\rightarrow$ câblage.

Il arrive qu'il y ait des trous dans la série par rapport à l'enchaînement des transformations théoriques possibles. Au point de vue de la génétique lexicale transformationnelle, ces maillons manquants ont une existence virtuelle. Ces trous constituent des appels à la création néologique. L'absence n'est qu'un accident historique à effet momentané : ainsi fonctionnaliser - seul enregistré par le Dictionnaire des mots nouveaux de Gilbert - appelle nécessairement fonctionnalisation qui a sans doute déjà été réalisé par quelque locuteur. Ou bien l'accident consiste dans la disparition d'un élément de la série et personne ne peut dire si elle sera momentanée, si le mot ne resurgira pas quelque jour comme un néologisme d'emploi ou si la disparition est définitive.

La série transformationnelle se fonde sur ces règles de transformation plus que sur la forme lexicale réalisée. Aussi peut-il arriver que le morphème base soit réalisé dans le lexique sous un aspect divergeant morphologiquement avec les termes de la série. Ainsi, dans le domaine spécial de la chimie, le terme combustion et sa série combustible, comburant impliquent une base verbale qui n'existe que sous la forme brîler.

On a vu que l'aptitude du morphème de base à générer une série lexicale transformationnelle est liée à sa classe syntaxique et à l'homogénéité sémantique de la série. On peut alors se demander comment se produit l'autonomisation sémantique d'un morphème de base par rapport à un homonyme morphologique. Cette différenciation ne peut s'expliquer par des règles purement syntaxiques et transformationnelles. La diversification du contenu sémantique relève de l'étude du mot sémantique et socio-linguistique. Tout au plus, peut-on noter que dans la chaîne des morphèmes lexicaux certaines classes se prêtent mieux à cette diversification en vertu de traits afférents à la classe. Ainsi le verbe exige syntagmatiquement la réalisation de l'agent de l'action et de l'objet de l'action s'il est transitif. Le substantif issu du verbe admet, par contre, l'effacement de l'agent et par conséquent la généralisation du contenu sémantique ou sa spécialisation, son émancipation par rapport à la phrase de base. Ainsi dans la série opposer (s'opposer) - opposition - oppositionnel, il est clair que le rapport de transformation - opposer $\rightarrow$ opposition - implique, dans le substantif dérivé, la présence de la personne qui s'oppose et de ce à quoi elle s'oppose - tandis que la relation opposition $\rightarrow$ oppositionnel se situe à partir d'un morphème - opposition spécialisé dans le domaine politique - la base ne contenant pas de référence à un agent, ni à un objet déterminé. La classe syntagmatique du substantif offre donc le maximum de possibilités de nouvelles lexicalisations par la diversification sémantique des emplois du morphème.

2.4. Le morphème-base, en vertu de son caractère essentiellement syntagmatique, se présente sous l'aspect du mot dans son intégralité morphologique de fonction- 
nement dans la phrase. C'est en effet le morphème tel qu'il est réalisé dans la phrase de base qui engendre le dérivé et non un groupement de phonèmes abstrait de cette forme réelle, appelé étymon dans la grammaire historique. Les règles morpho-phonologiques de jonction des éléments jouent de telle manière qu'il est souvent difficile de faire le départ entre le groupement phonématique dit base et le groupement afférent à l'affixe. Dans gestuel, la base est-elle gest ou gestu? Dans fuséologie, faut-il couper fuse - ologie - ou fuséologie ou fuse $o$ - logie? La relation de filiation s'établit à partir d'un morphème de base reconnu globalement comme appartenant à une classe syntagmatique pour aboutir à la substitution d'un autre morphème appartenant globalement à une nouvelle classe. Peu importe la délimitation exacte du groupement phonématique de référence à cette classe. C'est d'ailleurs ce qui explique que des éléments phonématiques puissent être arbitrairement détachés d'une souche pour servir de témoins d'une certaine classe (iser au lieu de $e r$ pour les verbes, tron dans le vocabulaire de la physique nucléaire). C'est aussi ce qui justifie la troncation de morphèmes base pour obtenir un nouveau terme par soudure gelule $<$ gel (arine) et ule $<$ de capsule. Ce principe de génération à partir de la globalité du mot fonctionnant dans la phrase a aussi pour conséquence d'effacer l'opposition traditionnellement établie entre la procédure de dérivation par affixe et la création par composition. Ce sont en réalité des transformations qui s'opèrent selon des modalités différentes dont le principe est également syntagmatique dans l'un et l'autre cas.

\subsection{LE MOT SÉMANTIQUE}

3.1.1. Morphème et sémème. - Définir le mot sémantique consiste à délimiter la zone de signification du signifiant qui n'est pas étroitement dépendante de la construction syntaxique, si l'on se place dans la perspective de la grammaire générative. Seuls les mots dits «mots grammaticaux » constituent des classes homogènes d'unités à fonction intrinsèquement syntaxique (prépositions, conjonctions, déterminants) ; ils sont considérés comme des opérateurs syntaxiques doués d'un contenu sémantique réduit, pour l'essentiel, à celui afférent à leur fonction. Le lexique proprement dit est formé de segments lexicaux qui remplissent pleinement à la fois la fonction de morphème lexical définie antérieurement et la fonction de signification. On pourrait donc dire que le mot sémantique est la somme des valeurs de signification du segment lexical qui ne sont pas uniquement dépendantes de sa fonction grammaticale et des restrictions de combinaison que celle-ci entraîne dans la constitution de la phrase. Pour reprendre notre exemple précédent du verbe brûler, il est morphème lexical seulement en tant que verbe intransitif ou transitif, trait syntaxique qui implique une certaine structure de phrase et en tant que porteur du sème «combustion», ce qui sélectionne parmi les lexèmes combinables avec lui ceux qui comportent le trait «combustible» ou le trait « agent de combustion».

La relation entre le syntaxique et le sémantique afférents à un même segment phonologique pose la question de la priorité d'un élément par rapport à l'autre. $\mathrm{Au}$ point de vue de la structure de la phrase, brûler transitif et brûler intransitif 
sont deux formes lexicales, homonymes mais différentes. Du point de vue du contenu sémantique essentiel ils sont un même lexème défini par les sèmes *action» et «combustion». Par-delà les réalisations syntaxiques diverses du segment lexical, le mot "sémantique » se fonde sur l'unité de la référence dans le système conceptuel ou dans l'expérience du monde. Le mot sémantique se définit par la constance de la correspondance entre un segment phonologique et un certain nombre de traits constitutifs d'un signifié qui forme le relais entre le langage d'une part, la pensée et l'expérience humaine, d'autre part. Ces traits sont généralement appelés sèmes et le faisceau qui les rassemble dans une unité de signification peut être appelé «sémème » selon la terminologie de $\mathrm{B}$. Pottier, de Coseriu et de Hegger. L'unité dans la référence est aussi indispensable à la communication entre les locuteurs que la règle de la construction syntaxique inhérente au morphème.

3.1.2. Les implications entre le sémantique et le syntaxique sont souvent si complexes qu'il est difficile d'isoler le morphème et le sémème. En principe, les sèmes d'un mot qui n'influent en aucune façon sur la structure syntaxique de l'énoncé doivent relever seulement du sémème. Il en est ainsi, par exemple, des traits qui définissent un sous-ensemble de «matière combustible » dans leur relation avec le verbe brûler. Ils forment une nomenclature des corps combustibles. Cependant dans le cadre d'une construction syntaxique du morphème résultant de traits sémantiques fondamentaux (brûler $=$ action + combustion) une variation sémantique affectant le sème principal peut se produire (combustion $=$ ardeur passionnelle). La structure syntaxique s'en trouve modifiée : le mari brûle de jalousie. Le sème "passion » ne peut apparaître qu'avec l'expression d'un complément du verbe intransitif se référant à une passion définie. Doit-on décider que le trait figuré est inclus dans le morphème parce qu'il se répercute nécessairement sur la structure syntaxique afin de rendre manifeste la mutation sémantique? Faut-il considérer, au contraire, ces transformations comme relevant seulement du sémème? Le débat est difficile à trancher.

3.1.3. Certains emplois métaphoriques constituent des sémèmes sans aucune implication syntaxique. Il s'agit de syntagmes à valeur figurée dont la signification réside, non dans la relation des éléments entre eux, mais dans la répétition de la séquence avec une signification différente de celle qui résulte des sèmes inclus dans chacun des termes constituants. La locution «mettre la charrue avant les bœufs $\gg=$ «intervertir l'ordre normal de succession de deux actions $\gg$ ne tire cette signification que de l'usage répandu dans la communauté linguistique et transmis de génération en génération.

L'isolement du sémème par rapport au morphème apparaît aussi avec évidence dans des unités complexes dont la signification est fondée sur l'unicité de la référence. Dans des unités du type sécurité sociale, ce ne sont pas les traits syntaxiques substantif et adjectif et la relation de détermination impliquée par eux, ni la compatibilité sémantique essentielle entre le trait «état d'esprit confiant» et le trait « relatif à la société » qui confèrent à la séquence lexicale sa fonction sémantique de référence à l'institution «sécurité sociale», mais bien l'expérience 
commune des avantages et des inconvénients de l'institution condensée dans l'usage de cette combinaison de morphèmes.

3.1.4. La fonction de signification de l'unité lexicale et l'autonomie fonctionnelle du sémème, par rapport au morphème, apparaît avec le plus d'évidence dans la synonymie, l'antonymie et, dans une moindre mesure, l'analogie.

Le synonyme, en effet, est un mot commutable avec un autre mot de telle façon que l'essentiel de la signification de l'ensemble syntaxique d'une phrase réalisée demeure identique. Si dans la phrase : il ne pouvait modérer le galop de son cheval, je substitue à cheval le lexème monture, le contenu de signification n'est pas essentiellement modifié ; seule la forme signifiante change. La commutation synonymique en effet s'opère dans le même cadre syntaxique du substantif. Les variations syntaxiques sont en général réduites au changement de genre, à la substitution d'une unité lexicale complexe à une unité simple ; ce qui permet, en définitive, la commutation, c'est une certaine identité du noyau de signification, indépendante à la fois de la forme signifiante et de la fonction syntaxique du lexème et qui ne peut se répérer qu'au niveau du sémème. Il en est de même de l'antonyme dont l'insertion dans la phrase s'opère sans mutation dans le schéma syntaxique. La modification consiste en une commutation de la forme signifiante, comme dans la synonymie, et en une inversion du contenu de la signification, définissable sur le seul plan du sémème. L'analogie, enfin, telle qu'elle apparaît dans les dictionnaires dits analogiques est une fonction sémantique hybride; en ce sens que le rapprochement établi entre des lexèmes se fonde, tantôt sur une approximation relative des traits de signification des sémèmes comme dans la synonymie, tantôt sur un lien d'association dans la réalité extra-linguistique. Ces différentes opérations ont pour trait commun, cependant, qu'elles concernent, dans le mot, le seul sémème et en démontrent l'autonomie de fonctionnement dans le langage.

\subsection{LE MOT RÉFÉRENTIEL}

3.2.1. Dans le mot référentiel qui a pour fonction de désigner, de dénommer les éléments de la réalité du monde réel ou conceptuel, les traits syntaxiques apparaissent comme nécessaires pour fonder l'insertion du mot dans l'enchaînement de la phrase, mais comme secondaires dans la définition du sens du mot. Celui-ci réside seulement dans la désignation de la chose ou du concept. Le mot référentiel a pour fonction de permettre la description de la structure des choses par leur énumération en les différenciant les unes des autres. Il est un terme de nomenclature. Il se définit par l'énumération des traits structurels de la chose désignée. Le mot référentiel se présente, en particulier, sous l'aspect du terme technique et scientifique qui revêt surtout la forme syntaxique du substantif convenant à la dénomination, à l'identification : la forme du verbe apparaît surtout pour désigner le procès de transformation de la chose ou de fabrication de l'objet.

3.2.2. Il serait exagéré, cependant, de refuser au mot technique ou scientifique la qualité de sémème à partir de cette fonction essentielle de dénomination. La dénomination implique certes l'univocité du terme, une certaine identification avec la chose et par conséquent un faisceau de sèmes stable, non soumis aux 
mutations sémantiques selon les locuteurs et selon l'insertion syntaxique. Mais le mot terminologique a aussi pour fonction de permettre la communication de l'expérience par la référence à un ensemble de sèmes qui le constituent comme unité linguistique. L'expérience varie selon les locuteurs. Si le mot fauteuil, dans la terminologie de l'ameublement, désigne un type d'objet qui s'oppose à chaise par la technique de fabrication, les matériaux employés, il est aussi le mot qui, pour les usagers, comporte le sens de «siège », de «confort», d' "aisance ». Le mot terminologique ne peut demeurer cantonné à un seul domaine de la réalité. Le système général de référence par le langage au monde et à l'expérience est fondé sur la disproportion inévitable entre l'infini des choses à désigner et le faible capital de signes disponibles pour cette désignation. Il s'ensuit que chaque signe, chaque nom tend à être utilisé dans plusieurs domaines différents. Le passage d'un domaine dans un autre s'opère en vertu de liens qui se situent dans l'expérience elle-même entre des techniques et des sciences apparentées. Le même mot prend des valeurs de référence différentes dans chaque domaine dont le cumul constitue le sémème du terme technique. Le même signifiant en effet, doté de la même combinatoire syntaxique, comporte des traits différents dans chaque domaine, mais aussi un minimum de traits communs à l'ensemble des emplois. $V o l$ en astronautique est différent de vol en aviation et de vol en ornithologie, mais ils comportent tous trois les traits « suspension et déplacement au-dessus de la terre $»$.

Le mot terminologique fonctionne comme sémème du fait qu'il est intégré à un ensemble de mots propres à un système linguistique. S'il en était autrement, un seul mot de référence pourrait être adopté dans toutes les langues en considération des seuls traits afférents à la chose ou au concept. Or, la transposition d'un terme technique d'une langue dans une autre soulève non seulement des problèmes phonologiques et grammaticaux mais aussi sémantiques.

Enfin, le mot terminologique, par sa référence même à la réalité objective, est entraîné dans toutes sortes de mutations. Il existe en effet des relations d'association conceptuelles entre les choses qui s'opèrent par l'intermédiaire des mots. Les apparentements établis sur le plan de la perception ou de la pratique sociale donnent lieu à des mutations sémantiques dans le contenu de désignation, notamment quand les mots techniques et scientifiques viennent à être employés par d'autres locuteurs que les savants ou les techniciens. C'est là une source essentielle de métaphorisation, si bien que le même signifiant cumule la référence dénominative et la valeur sémantique nouvelle. Le lexème est alors aussi pleinement un sémème qui assume à la fois la signification référentielle et la signification figurée.

\subsection{LE MOT DU DISCOURS}

L'entière signification du mot ne saurait être dégagée en dehors de sa réalisation en phrase de discours. Le contenu sémantique, en effet, se distribue entre la matrice lexicale au niveau de laquelle s'opèrent la catégorisation grammaticale et la distribution des sèmes essentiels qui conditionnent la séquence syntaxique, le sémème dont le fonctionnement implique une certaine structure 
sémantique générale de la référence à l'univers de la pensée et de l'expérience, et la phrase de l'énonciation où interviennent la personne du locuteur et la situation d'élocution.

La transformation du schéma de phrase en phrase réalisée suppose la communication entre un sujet parlant qui énonce la séquence syntaxique réelle et un auditeur présent ou fictif qui la reçoit. Chacun de ces êtres de chair et de sang est façonné psychologiquement et sociologiquement, animé de motivations particulières dans le dialogue; le langage apparaît dès lors comme l'instrument de communication d'êtres pensants, membres d'une communauté linguistique, dans une situation donnée. À ce stade, le problème qui nous concerne est de discerner quel contenu sémantique du mot résulte de cette activité langagière.

3.3.1. Le mot, unité de discours, cesse d'être une unité parmi la totalité du lexique de la langue pour devenir un élément de l'élocution d'un individu, un élément du vocabulaire qu'il a enregistré dans sa mémoire. C'est dire que sa définition comprend certes l'essentiel des sèmes qu'implique la structure générale de la signification issue d'une expérience commune, mais pas tous, et inégalement perçus par l'individu. Du fait qu'un mot est intégré au vocabulaire particulier d'un locuteur en l'absence d'autres, il entre dans de nouvelles relations. Certaines oppositions, certaines commutations qui lui donnaient son statut en langue n'opèrent plus dans la mesure où le terme nécessaire à la réalisation de cette opposition ou de cette communication n'existe pas dans le vocabulaire du sujet parlant. Tel locuteur n'aura à sa disposition que la variation synonymique cheval / canasson / bourrin, tandis que tel autre pourra user de la gamme cheval / monture /coursier / haridelle et tel locuteur paysan, de cheval / bidet, boulonnais, percheron, etc., et tel amateur de courses de chevaux, de pouliche, trotteur, sauteur, yearling. On voit les répercussions de cette différence numérique dans l'étendue du vocabulaire quant au type du discours et à la possibilité de nuancer l'expression. Le mot de discours se définit donc sémantiquement en partie par cet aspect quantitatif.

Le vocabulaire d'un locuteur doit s'analyser du point de vue de son étendue, selon plusieurs critères. Tous les mots qui interviennent dans la communication avec ses interlocuteurs ne lui appartiennent pas avec la même plénitude. Il y a d'abord ceux dont il use couramment et qui sont du langage de la plupart des interlocuteurs, ceux qui lui appartiennent en propre en vertu de son expérience personnelle ou de certains tics de parole ; il y a ceux qu'il ne fait que comprendre dans la bouche de l'interlocuteur, mais qui n'entrent pas dans son usage, qui appartiennent à l'élocution de l'autre; il y a ceux qu'il ne comprend pas mais qu'il parvient à interpréter en vertu d'une certaine connaissance du système de la langue; il y a enfin ceux dont il ne peut que dire qu'ils sont de sa langue par la sonorité. En définitive, le vocabulaire d'un locuteur se définit par certaines interférences avec le vocabulaire des autres, mais le vocabulaire de chacun d'eux, s'il comporte un noyau commun, se différencie par des zones qui définissent sa personnalité langagière. Le volume du vocabulaire de chaque locuteur se modifie dans la succession des communications; il apprend constamment des mots nouveaux, il en adopte de préférence certains par imitation de l'interlocuteur en 
fonction du prestige que celui-ci exerce sur lui et en oublie d'autres en fonction de l'évolution de ses facultés intellectuelles.

3.3.2. Comment se constitue le capital de mots qui entrent dans l'idiolecte d'un sujet parlant? L'étendue de son vocabulaire est fonction d'une série de facteurs physiologiques (âge et sexe), sociologiques (milieu familial, éducation et culture, mode de vie, relations, région, métier, classe sociale). Chacun de ces facteurs, auxquels il faudrait sans doute ajouter beaucoup d'autres et notamment la situation de bilinguisme, constitue un faisceau spécifique correspondant à la structure idéologique et linguistique de sa personnalité de sujet parlant. Leur description relève de la socio-linguistique. En outre, le locuteur ne mobilise pas tout son capital de vocabulaire dans chaque situation de locution. Pour peu qu'il dispose d'une gamme un peu étendue, il a enregistré des termes qui ne correspondent pas à son type social d'élocution, mais dont il est capable d'user selon la personnalité de l'interlocuteur pour créer chez lui une réaction de confiance. Cette technique de l'adaptation à l'autre est bien connue des spécialistes de l'interview et du langage publicitaire. Chaque conversation se situe donc dans un certain climat psychologique qui conditionne le choix des termes employés. Des interdits moraux et sociaux pèsent sur de nombreuses situations d'élocution. L'étude des nombreuses implications de la psychologie des locuteurs dans leur élocution appartient à la psycho-linguistique. La spécificité du vocabulaire du sujet parlant ne peut, en définitive, être appréciée convenablement que dans un corpus d'énoncés produits (écrits ou oraux) parce que les variables d'élocution ont été ainsi à peu près circonscrites.

Les variables qui déterminent le choix des mots dans le discours produit peuvent être d'ordre géographique. À l'intérieur d'une même langue, surtout quand elle s'est constituée dans une longue histoire, il existe des parlers régionaux, des dialectes, qui ne sont jamais totalement éteints, même quand une langue commune s'est imposée. Le mot régional est vivant, quand il correspond à des réalités régionales, géographiques ou ethnologiques (mas, aïoli en Provence). L'option dialectale s'impose par la permanence de désignations spécifiques à l'intérieur de certaines professions (la pièce noire de la boucherie marseillaise au lieu de la tranche dans la boucherie parisienne). Le locuteur se trouve ainsi dans une situation de bilinguisme lexical. Une seconde catégorie de variables est d'ordre socioculturel. Il n'est certes pas possible d'affirmer qu'il existe autant de vocabulaires que de classes sociales. Cependant, dans la mesure où la culture est distribuée, dans certains types de société, en fonction de la classe sociale, le vocabulaire disponible pour chaque locuteur varie selon la classe à laquelle il appartient. Des groupements sociaux interfèrent avec la distribution en classes selon les activités sociales et professionnelles. On peut distinguer un niveau de vocabulaire moyen employé dans la communication interclasses et intergroupes, un niveau cultivé, un niveau argotique et populaire. Ces niveaux ne se trouvent d'ailleurs pas souvent réalisés d'une façon homogène dans le discours des locuteurs, sauf l'argot cohérent qui constitue un double du vocabulaire normal de la langue.

3.3.3. Une autre série de variables dans la distribution des mots est formée par le type de discours. Il se détermine en fonction de la situation d'élocution et de 
l'intention de signification du locuteur par rapport au récepteur. Certes le discours se définit par la totalité des composantes de l'expression linguistique, l'intonation, la structure syntaxique de la phrase, mais surtout par l'usage des mots. On peut distinguer le discours solennel, le discours familier, le discours didactique, le discours politique, religieux, etc. L'étude de tous ces aspects relève de la rhétorique ou encore de l'analyse du discours qui étudie le lien entre le type de phrase et le vocabulaire qui entre dans le discours en fonction de la situation et de la personne du locuteur. La situation d'élocution est souvent liée à l'objet d'étude. Le discours et son vocabulaire se déterminent alors selon la spécialité. Des études ont été menées en particulier sur la relation entre les sciences et le vocabulaire du discours tenu à propos d'elles, par exemple le vocabulaire général d'orientation scientifique et plus précisément sur une science et le vocabulaire du discours qu'elle suscite dans les textes qui l'exposent (Dictionnaire contextuel de géologie de J. L. Descamps, à paraître)

3.3.4. On doit faire une place privilégiée à la variable de discours écrit que représente la littérature; elle répond à un type de communication particulier : l'interlocuteur est absent et anonyme; le locuteur n'est nullement entravé dans le choix des mots, par lesquels il tend à affirmer sa personnalité, son originalité, autant qu'à communiquer sa pensée. Son message prend différentes formes selon les genres, la poésie ou la prose. L'utilisation de toutes les ressources du vocabulaire est la fonction même de l'écrivain. Il n'est pas étonnant, dès lors, que dans son élocution peuvent apparaître aussi bien des mots vieillis dont l'usage ne se perpétue que par les textes littéraires que des mots nouveaux spécialement forgés par l'auteur selon les nécessités de son expression.

3.3.5. Dans le lexique d'une langue apparaît, en dehors d'un noyau permanent qui résiste à l'usure des siècles et qui correspond aux nécessités primordiales de toute communication, une certaine stratification des mots résultant du dépôt laissé par chaque génération de locuteurs. Dans un état de langue coexistent des mots vieillis, encore employés par les hommes de la génération la plus ancienne, les mots communs aux différentes générations vivantes et les mots qui s'installent dans l'usage de la génération la plus jeune en particulier. Les mots déjà disparus survivent néanmoins par le moyen de la tradition culturelle, si bien qu'ils peuvent réapparaître dans le langage des personnes cultivées, en particulier chez les écrivains qui se plaisent à ressusciter des mots de l'ancienne langue. Il faudrait encore ajouter les mots qui se chargent d'une valeur symbolique dans l'histoire des peuples, dans la vie des sociétés et dont le prestige s'accroît, demeure ou s'éteint selon les étapes de la transformation de ces sociétés. La diversité de la valeur sémantique des mots du discours semble bien difficile à cerner par une théorie qui tienne compte de toutes les variables dans la production du discours et dans la réalisation des lexèmes de la phrase. On peut, sans doute, établir qu'un discours spécial s'analyse selon une structure de phrase à laquelle est lié un certain type de vocabulaire, en vertu du principe unique de production de la phrase et de la génération de mots construits; mais il faudrait aussi pouvoir vérifier si dans le discours d'un locuteur moyen, disposant d'une certaine gamme de vocabulaire, la substitution d'un terme plus familier à un terme plus relevé, par exemple causer 
au lieu de s'entretenir avec, est liée au processus de formation de la phrase, ou bien à une impulsion du locuteur à partir de motivations incontrôlables. L'inclusion du trait populaire ou argotique dans la matrice lexicale de la phrase ne semble s'imposer que pour les locuteurs qui ne possèdent qu'un seul registre de vocabulaire. C'est donc dans le cadre de chaque idiolecte que la question peut être tranchée. $\mathrm{Ou}$ bien il faut concevoir une sorte de langue neutre, une langue fonctionnelle du locuteur moyen, une norme lexicale par rapport à laquelle les variations apparaîtraient comme des écarts.

L'idéal d'une langue purement mathématique ou logique où chaque terme aurait une valeur univoque à jamais fixée et invariable dans toutes les situations ne peut se concevoir dans une civilisation développée, jouissant d'une langue chargée d'histoire, ni sans doute dans aucune langue. La forme signifiante, répondant à une certaine combinaison de phonèmes incluse dans la combinatoire phonématique du système linguistique, revêt nécessairement des valeurs diversifiées dans les divers idiolectes des sujets parlants, ou des groupes sociaux. Tout au plus, le mot terminologique peut-il garder sa pureté sémantique quand il fonctionne avec sa valeur référentielle dans un groupe homogène de locuteurs. Mais dès qu'il se répand dans l'ensemble de la communauté linguistique, il devient sujet à toutes les diversifications sémantiques. La polysémic est la règle de fonctionnement du langage. Chaque lexème ne peut dès lors être défini et décrit qu'à titre de morphème, de matrice lexicale incluant les traits syntaxiques et sémantiques les plus généraux. La diversité de ses réalisations sémantiques dans le discours peut ensuite être enserrée dans des schémas de description, mais ils sont arbitrairement construits et non issus du fonctionnement linguistique du terme.

\section{LE MOT MÉTALINGUISTIQUE}

Le mot, en tant que morphème lexical et sémème, est un élément de la production de la phrase et ne peut être dissocié d'elle. Mais il est aussi perçu comme une entité autonome en tant que signe linguistique en vertu d'une analyse qui isole ses constituants (signifiant, signifié, référent). Cette interprétation du langage produit donne lieu au métalangage, c'est-à-dire à un langage à propos des phrases engendrées par un locuteur dans une situation donnée.

4.1. On remarquera d'abord que, dans le métadiscours, ce ne sont pas seulement les unités lexicales appelées mots, c'est-à-dire les segments reconnaissables graphiquement par le blanc qui les séparent, qui peuvent devenir mots métalinguistiques, mais n'importe quel segment phonique ou graphique - aussi bien l'unité minimale appelée mérisme par Benveniste, le phonème, le morphème, le lexème, le syntagme et la phrase tout entière. Il suffit de transformer le segment isolé de la phrase de discours ou du mot en élément d'une nouvelle phrase en l'enserrant dans la catégorie grammaticale du substantif (votre s est mal formé; vous avez employé l'imparfait «ils sortaient», etc.). La phrase de métalangage implique une citation de l'élément extrait de la phrase de référence grâce à une marque quelconque, des guillemets pour le mot graphique, une pause avant le mot prononcé, un mot catégoriel d'introduction (l'imparfait ils sortaient, la lettre $s$ ) ou simplement un déterminant $l e$, un. Le lexème cité en métalangage ne doit pas être confondu avec 
le mot entre guillemets dans la phrase de discours. Il peut arriver, en effet, et notamment dans le discours journalistique, qu'un locuteur emploie un terme repris d'un discours réalisé sans l'assumer entièrement; il le met entre guillemets. Cette modalité d'emploi ne le mue pas en mot métalinguistique car le mot ainsi inséré dans la phrase remplit sa fonction syntaxique, la marque des guillemets signifiant seulement la volonté de distanciation par rapport au responsable du mot originel.

4.2. Il est utile aussi de faire une distinction entre le mot métalinguistique et le mot du stock lexical propre à un individu parlant. Les termes que celui-ci connaît sont, en effet, disponibles dans sa mémoire en fonction de sa connaissance du lexique; ils y ont été enregistrés après avoir été perçus, remarqués et interprétés dans des phrases de discours. Ils ne sont pas encore des mots «linguistiques» puisqu'ils ne sont pas intégrés à des phrases réelles prononcées en situation selon les règles de la construction syntaxique, quoiqu'il arrive souvent qu'ils soient enregistrés dans la mémoire comme éléments de phrases stéréotypées, sous forme d'unités phraséologiques. Ces mots de la mémoire individuelle doivent être considérés comme des matrices lexicales; ils sont réalisés ensuite dans l'énonciation par le locuteur en l'absence de tout jugement métalinguistique. La mémoire des mots propres au sujet parlant est différente de la mémoire collective représentée par le lexique que dresse le lexicographe en vertu d'une différenciation faite dans la totalité du lexique. Pour que le mot métalinguistique existe, il faut qu'au moins une de ces deux conditions soit réalisée, que le mot soit extrait d'un discours réel, qu'il fasse l'objet d'une nouvelle phrase dans un discours à propos du premier, impliquant un jugement et une assertion. C'est pourquoi les éléments de phrases tels que les syntagmes lexicalisés (machine à laver la vaisselle) ou même des phrases entières (qui vive; décrochez-moi ça) qui s'emploient comme mots ne sauraient être considérés comme des mots métalinguistiques; ils constituent seulement des stéréotypes qui ont été convertis en unités lexicales par la répétition et l'enregistrement dans la mémoire collective.

4.3. Les mots métalinguistiques abondent chaque fois qu'un discours est produit systématiquement à propos d'un autre discours. C'est le cas dans la pédagogie d'une langue. L'exercice connu sous le nom d'explication de textes consiste précisément, pour une bonne part, à extraire les mots d'un texte pour porter un jugement sur eux; il en est de même dans l'enseignement de la grammaire, de l'orthographe, dans la critique littéraire traditionnelle qui comporte des jugements sur des fragments de l'œuvre d'un auteur et son vocabulaire, seulement occasionnellement dans la nouvelle critique qui vise essentiellement à une réinterprétation de l'œuvre et à la production d'un discours parallèle à celui de l'auteur jugé.

4.4. La lexicologie quantitative qui vise à fournir un instrument d'analyse du langage réellement produit commence par extraire les mots du texte pour les interpréter linguistiquement à partir de données quantitatives. Le problème essentiel est même de découper le texte en unités démontrables, de réduire les mots syntaxiques de la phrase réelle en mots métalinguistiques, en faisant abstraction de toutes les relations synonymiques, homonymiques pour ne prendre en considération que l'unité numérique. Elle est ainsi conduite à ne retenir que le critère le plus externe à la fonction linguistique, l'unité du signifiant établie par la graphie 
séparée des segments lexicaux. La liste des unités s'établit, en définitive, par un jugement porté à propos de chaque mot du texte, du type «m. est une unité quantitative $\gg$ qui le transforme en mot métalinguistique.

4.5. Les deux procédures ci-dessus évoquées constituent des métadiscours à partir des mots d'un texte réellement produit. En lexicographie, la situation du locuteur, c'est-à-dire du lexicographe, est beaucoup plus complexe, comme l'a montré. J. Rey-Debove dans son ouvrage. Il existe, en effet, plusieurs types de dictionnaires. Les simples glossaires sont établis à partir d'un corpus de textes nettement définis ; l'acte qui consiste à dresser les mots d'un glossaire correspond à la procédure de transformation d'un mot « linguistique » en mot métalinguistique, en vertu d'un jugement «tous les mots de cette liste figurent dans ces textes». Dans les formules de dictionnaires dits contextuels tous les termes de la nomenclature sont extraits d'un corpus de textes et ils donnent lieu à une explication génétique et sémantique de leur contenu. Mais dans les dictionnaires de grande dimension, les mots de la nomenclature ne sont pas choisis seulement en raison de leur appartenance à un corpus de textes limité. Chaque lexicographe établit une liste d'œuvres d'auteurs d'où sont extraits les exemples produits à l'appui des définitions, possède un fichier d'exemples; il arrive cependant souvent que tel emploi d'un mot soit défini par référence, non pas à un mot réalisé dans une phrase, mais à un emploi mentionné déjà dans un autre dictionnaire. Mais, surtout, l'établissement de la nomenclature d'un grand dictionnaire de langue exclut la procédure qui consisterait à partir d'un rassemblement préalable d'un corpus de textes produits pour en extraire tous les mots figurant effectivement dans ces textes. Le repérage de l'énorme quantité de mots du lexique de la langue rassemblés dans un grand dictionnaire ne peut se faire qu'en prenant pour base des dictionnaires antérieurs. L'inclusion d'un mot dans la nomenclature du nouveau dictionnaire en fait un mot métalinguistique au second degré pourrait-on dire. L'acceptation ou le rejet de la nomenclature d'un mot implique un jugement de valeur sur le mot, sur son appartenance au lexique de la langue définie selon un certain modèle, sur le degré de vieillissement, ou le degré d'installation dans l'usage. Le mot de dictionnaire apparaît donc par excellence comme un mot métalinguistique par la procédure du choix de la nomenclature. Il l'est davantage encore par l'établissement de l'article du dictionnaire qui consiste essentiellement à faire du mot-entrée le sujet d'une série de phrases qui définissent son contenu sémantique et ses différentes valeurs d'emploi.

4.6. Enfin le mot métalinguistique est l'objet même de la science de la linguistique. Le fonctionnement des mots du lexique constitue une branche de la linguistique, la lexicologie. II convient de distinguer les techniques d'analyse qui s'appuient sur un corpus de textes produits comme dans la linguistique structurale classique et celles qui prennent pour appui des phrases générées théoriquement en éliminant les variables des phrases produites en situation par des locuteurs réels, comme dans la grammaire générative. Le raisonnement établi à partir des termes de ces phrases syntaxiquement grammaticales ou réellement produites, transforme les éléments lexicaux en mots métalinguistiques. Dans les dictionnaires de linguistique qui prennent pour objet les termes mêmes de la linguistique, ce sont les 
termes de la nomenclature de cette science qui passent du statut linguistique d'éléments de la phrase chez les auteurs de diverses théories au statut métalinguistique.

\section{LEXIQUE ET PRODUCTIVITÉ}

5.1. La définition du mot dans ses différentes fonctions linguistiques nous conduit à constater qu'il est une réalité linguistique protéiforme, qu'il apparaît sous l'aspect d'une matrice lexicale dont la productivité ne saurait être fixée ni arrêtée, sous l'aspect d'un pôle de fixation d'une infinité de valeurs sémantiques par rapport au locuteur, au milieu social, et à sa situation d'élocution. Comment définir, dans ces conditions, le lexique d'une langue autrement que par la totalité des unités et des emplois lexicaux qui ont existé, commencent à cesser d'exister, existent ou commencent à exister dans la communauté linguistique, c'est-à-dire chez tous les individus de cette communauté, dans tous les milieux et groupes sociaux pour exprimer toutes les choses du monde, de la pensée, tous les faits de la civilisation, toutes les motivations et les nuances d'expression en fonction de la vie psychologique et sociale de tous ces locuteurs? C'est dire que le lexique ne saurait être limité, pas plus que le mouvement de la vie, de la pensée, de l'histoire. Le lexique, dans ces conditions, ne constitue pas un ensemble fini, comme l'ont remarqué les structuralistes, en l'opposant au système clos des phonèmes et des morphèmes; les tentatives pour en donner une description exhaustive en tant qu'ensemble clos sont donc vouées à l'échec. On ne peut embrasser cette réalité mouvante que dans la perspective d'une théorie de la création continue. On la trouve dans la grammaire générative et transformationnelle qui ne traite pas le lexique comme un secteur autonome de la langue, mais le fait procéder de l'activité créatrice du locuteur par la phrase et ses diverses transformations (cf. Jean Dubois, la Phrase et ses transformations).

Nous disposons maintenant, grâce à la publication du Dictionnaire des mots nouveaux de P. Gilbert (Hachette-Tchou, 1972), d'un instrument de vérification de cette théorie, indépendamment des positions théoriques de l'auteur. Son dictionnaire représente la description d'un moment de la création lexicale dans la communauté linguistique française hexagonale. Il permet de vérifier quels sont les grands axes de la productivité lexicale dans la période contemporaine.

5.2. Pour donner une idée de cette productivité, nous nous guiderons sur l'analyse du mot que nous avons faite, et distinguerons celle du morphème en tant que matrice lexicale et celle du sémème sous l'aspect du mot référentiel et du mot de discours.

Nous avons décrit par ailleurs les structures syntactico-lexicales qui rendent compte du mécanisme de production des termes construits à partir de la transformation d'une phrase de base et nous nous permettrons d'y renvoyer (Grand Larousse de la langue française, vol. I, Introduction). Nous constatons qu'une grande partie des néologismes cités par P. Gilbert relèvent de ces processus de verbalisation, nominalisation, adjectivation. On peut observer qu'en ce qui concerne la création suffixale, la productivité de tel morphème ne se manifeste pas seulement dans une catégorie grammaticale mais dans deux ou même trois en vertu 
d'une chaîne de dérivations (-iser -isation -isant / -able -abilité), que souvent le maillon de la chaîne de dérivation qui manque est implicite dans la première création réalisée, soit que, par exemple, la formation en iser est antérieurement réalisée et entraîne celle en isation, soit que la formation première en isation implique la création ultérieure au verbe (vedettisation $\rightarrow$ vedettiser $*$ ). L'explication de la productivité de certains suffixes repose donc sur la productivité du schéma syntaxique de production. Mais il apparaît en outre que, dans le cadre de ce schéma, certaines formes suffixales sont plus particulièrement productives. Cette productivité est alors d'ordre paradigmatique et sociologique. Le succès d'un élément lexical tient à l'accumulation des créations déjà produites qui retentit par un phénomène de répétition, de locuteur à locuteur et de groupe à groupe, pour s'étendre à la collectivité tout entière, comme l'a montré J. Peytard dans sa thèse sur la préfixation. On relève parmi les suffixes les plus dynamiques : suffixes de nominalisation : isme, iste, ité, age, tique, ticien; suffixes d'adjectivation : el (tionnel), iel, uel, al, ien (comme suffixes de dérivation des noms propres) ; suffixes de verbalisation : iser, ifier. Il faudrait ajouter d'après les relevés faits par Bengt Hasselrot les suffixes de diminutif nominal ette et de diminutif verbal oter, ouiller (Étude sur la vitalité de la formation diminutive française au $X X^{e}$ siècle, Uppsala, 1972).

Selon notre conception de la création lexicale, la procédure de la préfixation et de la composition, comme celle de la suffixation, ont comme point de départ le schéma syntaxique d'une phrase de base. Nous relevons parmi les préfixes productifs de forme française, latine ou grecque, issus d'un adverbe ou d'une préposition a (an), après, avant, archi, contre, ex, extra, hyper, in, infra, inter, non, post, pré, pro, quasi, sans, semi, sous, sub, super, sur, trans, ultra, et particulièrement les éléments de modalisation sémantique du procès du verbe de et re. Les procédures de composition les plus dynamiques qui apparaissent dans le Dictionnaire des mots nouveaux sont celles qui reposent sur la combinaison syntaxique de deux noms ou d'un nom et d'un adjectif. Celle-ci se réalise sous deux formes : ou bien par la juxtaposition selon une transformation lexicale marquée par un trait d'union impliquant le plus souvent l'effacement du joncteurpréposition (plateau-repas, armoire-rangement), ou bien sous la forme du syntagme de phrase (banc d'essai, centre commercial). La polifération de ce type de composition se manifeste aussi sur le plan paradigmatique par la transformation de l'un des éléments en base d'une série, le plus souvent comme second élément (-miniature, -de pointe) mais aussi comme premier élément (idée- opération-). Le syntagme résulte souvent de la transposition lexicale d'un syntagme de phrase où l'effet du poids social de la répétition et de la fonction référentielle semble prédominer sur le schéma syntaxique de réalisation (plein emploi, point chaud, rectangle blanc). La nominalisation par composition se réalise très souvent sous la forme de la composition de type gréco-latin par la combinaison de deux éléments nominaux (neuro-chirurgie) ou d'un adjectif et d'un nom (monobloc) avec les mêmes phénomènes de prolifération d'un élément (mini, maxi, néo). Enfin, on voit fleurir le type de composition nominale par fusion de deux éléments issus par troncation de deux noms (diathèque $<$ diapositive et thèque). Une combinaison issue d'un syntagme verbal (verbe + nom) se révèle aussi très 
productive, sous la forme syntaxique française (chauffe-biberon) ou sous la forme syntaxique empruntée aux langues anciennes (ecocide). La combinaison adjectivale revêt le plus souvent la forme dite savante (socio-culturel). Il serait sans doute suggestif d'établir la comparaison entre la productivité de ces différentes formes lexicales et notamment de déterminer si, en définitive, les créations par préfixation et composition ne l'emportent pas en nombre sur les créations par suffixation dans la procédure de nominalisation tout ou moins. Quoi qu'il en soit, il apparaît que certains schémas de création lexicale sont d'une productivité permanente dans le français contemporain et qu'ils suscitent une production massive. Le directeur du Trésor de la langue française a avancé le chiffre de 3000 mots nouveaux par an, dans l'Express (cité par Hasselrot dans Étude sur la vitalité de la formation diminutive française au $X X^{e}$ siècle). Ce qui nous importe, à ce stade de notre raisonnement, c'est l'ampleur du chiffre en liaison avec le témoignage de productivité constitué par le Dictionnaire des mots nouveaux et les indications qu'il comporte sur les voies de cette productivité. Il faut encore préciser que ces témoignages ne concernent que les créations dans la langue écrite. Combien de mots nouveaux pourrait-on enregistrer dans les multiples conversations des locuteurs d'une communauté ? Nul ne le sait.

5.3. La production lexicale est sans doute plus importante encore en ce qui concerne la valeur sémantique des signes lexicaux. Chaque signe, comme on l'a vu, est voué à la polysémie par la multitude des choses et des concepts créés dans le domaine scientifique et technique et qui doivent recevoir une dénomination et par la multitude des désignations qui accompagnent toute évolution psychologique et sociologique. Pour ce qui est des choses nouvelles, il suffit d'évoquer le chiffre cité par P. Gilbert dans son introduction : 45000 mots nouveaux par an enregistrés par l'Institut national de la propriété industrielle désignant des marques déposées pour évaluer l'ampleur du phénomène. Les mutations constantes dans le comportement des groupes sociaux et des individus donnent lieu à des emplois nouveaux de mots existants dans une proportion incontrôlable, repérable seulcment quand ils s'étendent dans une certaine sphère de locuteurs. La création sémantique par métaphorisation suscite des emplois imprévisibles chez chaque locuteur, dans la mesure où elle se fonde sur une relation purement connotative établie par l'individu entre deux choses ou deux situations. Cependant la translation des signifiants d'un champ sémantique à un autre s'opère selon certaines constantes qu'il est possible de constater. Les termes d'une spécialité passent dans une autre spécialité qui lui est apparentée sur le plan de l'expérience humaine (de l'aviation à l'astronautique). Les termes des vocabulaires spéciaux deviennent des bases de création métaphorique lorsqu'ils appartiennent à des techniques, à des sciences connues expérimentalement par la masse des membres d'une société (les termes de l'automobile démarrer, déraper, etc.), de l'aviation (en catastrophe, descendre en flammes, etc.), du sport (décontracté, musclé). Les termes du vocabulaire commun se spécialisent dans un champ sémantique particulier (récupérer dans le domaine politique). Chaque base métaphorique peut devenir à son tour matrice lexicale (récupérer, récupération, récupérateur, récupérable) ce qui démultiplie l'effet de création. Et parmi les locuteurs, il faut réserver une place spéciale dans la création métaphorique aux spécialistes du verbe, les littérateurs, dont la langue 
se définit le plus souvent par un emploi de mots différents de ceux du locuteur moyen.

\section{DICTIONNAIRE ET NORME}

De notre définition du mot métalinguistique, il ressort que le dictionnaire monolingue est un ouvrage métalinguistique sur le lexique d'une langue. Il se fait à partir du fonctionnement du lexique, donc hors du lexique lui-même. C'est pourquoi il conviendrait d'être plus rigoureux dans la terminologie et de réserver le terme lexique à la désignation de la globalité des mots fonctionnant dans le langage, et le terme dictionnaire aux différents recueils de mots. Faire un dictionnaire c'est d'abord évaluer l'ampleur de l'ouvrage à réaliser et en déterminer la destination, opérer un choix dans le lexique, conférer aux mots choisis dans la masse lexicale le statut spécial de «mot de dictionnaire», construire un schéma de description et $\mathrm{y}$ insérer la matière sémantique vivante du lexique.

6.1. L'établissement de la nomenclature du dictionnaire, du corpus des éléments lexicaux à insérer constitue le premier acte de l'attitude métalinguistique par rapport au lexique de la langue. Le choix du lexicographe est commandé par la visée de sa description : le dictionnaire peut rassembler les mots référentiels de l'expérience de tel groupe social dans une activité scientifique ou technique (formule du dictionnaire technique), les mots dans un aspect particulier de leur fonction sémantique (dictionnaire de synonymes, d'antonymes, analogiques, de racines), les mots les plus fréquents (dictionnaire fondamental ou essentiel), les mots d'une époque (dictionnaire classique, de l'ancien français), les mots rares (dictionnaire des mots sauvages), les mots nouveaux (dictionnaire de néologismes), les mots d'un emploi difficile dans la phrase (dictionnaire des difficultés), sans compter les dictionnaires et glossaires d'auteurs. Si sa visée est plus globale, en rassemblant le plus de mots possibles dans leurs fonctions diverses, alors se présente l'option entre la formule encyclopédique (dictionnaire de mots référentiels avec discours didactique sur l'univers des choses et des hommes), la formule dictionnaire de langue (dictionnaire de morphèmes lexicaux et de sémènes dans leur fonctionnement en discours), ou la formule hybride linguistico-encyclopédique. Cette visée globale elle-même ne peut conduire à l'exhaustivité parce qu'il s'agit d'opérer un choix dans le continuum diachronique du lexique.

La seconde procédure métalinguistique consiste à donner au mot la morphologie arbitraire de mot de dictionnaire. Le mot-entrée se présente sous la forme du mot graphique délimité selon les règles typographiques, séquence phonématique séparée à droite et à gauche par un blanc. L'unité lexicale complexe ne figure que si les éléments composants sont réunis par un trait d'union. La catégorie grammaticale du mot est traduite arbitrairement, selon 1a tradition : infinitif du verbe, masculin de l'adjectif. La polysémie du mot se résout soit par la juxtaposition de morphèmes homonymes en tant qu'entrées différentes, soit par l'intégration de sémènes différents sous une entrée-vedette, selon la perspective étymologique diachronique ou synchronique du lexicographe. L'analyse sémantique est subordonnée à la catégorisation syntaxique ou à la filiation étymologique. 
La troisième procédure métalinguistique consiste à délimiter le contenu sémantique. Elle implique un jugement sur la distribution des sèmes dans les différents emplois et un classement qui repose sur une construction du lexicographe. Le nombre de divisions opérées repose en définitive sur son aptitude à procéder à une analyse fondée sur les critères offerts par la science de la linguistique ou sur une intuition d'usager de la langue, notamment quand il s'agit d'accorder ou non aux emplois métaphoriques le statut de sous-sémèmes.

La quatrième procédure métalinguistique résulte de la phrase de définition qui établit une équivalence entre un morphème lexical ou un sémème et une phrase affirmative dont le prédicat est constitué essentiellement par un mot substitut à contenu sémantique englobant (action, qualité, manière). Le caractère métalinguistique d'une telle phrase est illustré par le recours à la phrase réellement produite par un locuteur pour illustrer le fonctionnement linguistique du mot.

6.2. Le dictionnaire, ouvrage métalinguistique, a une fonction socio-linguistique. Il contribue à dégager une norme dans le fonctionnement du lexique de la langue. Il existe en effet deux aspects de la norme, soit qu'on la considère comme l'expression même de l'activité et de la productivité linguistique conformément aux règles du système d'une langue, soit qu'on la considère comme une pression de la communauté linguistique pour définir un certain usage des règles du système linguistique (cf. notre article, "Peut-on définir un concept de norme lexicale?» dans Langue française, à paraître). Le mouvement de vieillissement et de création inhérent au fonctionnement de la langue, et du lexique en particulier, les différenciations entre les locuteurs impliquent une intervention de la communauté pour déterminer un certain usage afin d'assurer l'intercompréhension et l'efficacité de la communication. La fonction du dictionnaire est de signaler ce qui est vieilli, de cataloguer les mots selon leur niveau afin de permettre une communication nuancée entre les membres de la communauté, de témoigner de la diffusion suffisante des mots nouveaux. Le dictionnaire est un instrument d'apprentissage des mots de la langue et de normalisation de leur emploi.

Mais l'établissement de cette norme socio-linguistique ne peut être indépendant d'un certain modèle social. La langue fonctionnelle de référence, au-delà des règles fondamentales de système, est nécessairement celle d'une partie privilégiée de la communauté linguistique. Car les locuteurs sont aussi des êtres sociaux par d'autres aspects que l'activité linguistique. Ils se répartissent en classes sociales, en groupes sociaux. Le destin de la communauté, sous tous ses aspects, repose, dans une période donnée, sur une classe dominante qui fournit le modèle linguistique. C'est pourquoi le dictionnaire relève aussi d'une certaine norme sociale, issue de la vision que la classe dominante a du lexique de la langue de la communauté. C'est en fonction de cette norme culturelle que se décident l'inclusion des termes nouveaux, selon une attitude puriste ou anti-puriste, celle des mots dits bas en vertu des règles de l'éducation, celle des mots empruntés aux langues étrangères, le choix des auteurs de référence dont la cote dans la communauté est déterminée souvent par l'appareil culturel de la société. Le lexicographe, luimême esclave des règles de sa discipline, n'est souvent pas en état de prendre la distance nécessaire pour se rendre compte de la sélection qu'il opère et de la responsabilité qu'il assume en contribuant à créer la norme lexicale. 
6.3. Le dictionnaire enfin est un moyen d'affirmation de la communauté linguistique dans la mesure même où il constitue une description des aspects essentiels du lexique global dont elle use. Dans une société non dangereusement exposée à la contamination culturelle et linguistique par une autre civilisation, ce dictionnaire consacre un certain nombre de désignations de concepts et de choses qui définissent les modes de penser et les activités sociales dans le cadre de cette société. Il acquiert une fonction didactique, en offrant le recueil des mots indispensables aux différentes formes et nuances de la communication entre les membres de la société ; il aide à maintenir un certain niveau de compréhension entre eux et entre les différentes générations. Dans les sociétés où règne le bilinguisme, ce dictionnaire est un tableau du lexique à maintenir pour assurer la survie du système linguistique propre à la communauté minoritaire; mais étant aussi le reflet du lexique réel, il est nécessairement une certaine description du lexique tel qu'il fonctionne réellement, tel qu'il résulte du jeu des langues en contact.

LOUIS GUILBERT

\section{QUESTIONS}

M. Rey : Je ne vais pas intervenir sur le problème des dictionnaires pour une raison simple, e'est que je suis absolument d'accord en tout ce qu'a dit $M$. Guilbert. Je voudrais faire une remarque sur la structure générale de son exposé qui m'a paru extrêmement intéressant. En allant de l'aspect lexème du mot à l'aspect mot de discours, en passant par le concept du sémème, c'est-à-dire la sémantique analytique, en passant par le concept de référence, aire de sémantique extensionnelle, on définit en effet une chaîne qui va du plus ou moins régulier, du plus ou moins étudié, du plus ou moins connu, du plus ou moins linguistique. Lorsqu'on s'en va dans la direction de la référence, c'est-à-dire vers la direction du mot de discours, on pose tous les problèmes qui me semblent être fondamentaux, parce qu'ils doivent être réinsérés dans la lexicologie, et qui sont ceux de la socio-linguistique, de l'ethno-linguistique, de la psycho-linguistique, de la théorie de la nomination, de la philosophie du langage, etc. C'est vers le début, vers l'aspect lexème, c'est-à-dire en fait vers la morpho-sémantique, puisque c'est de cela qu'il s'agit, que les régularités sont les plus grandes et que l'étude lexicologique est la plus rentable. L'utilisation de la grammaire générative transformationnelle en matière de morpho-syntaxe, c'est évidemment une chose possible et une chose qu'on doit approfondir sérieusement parce que les travaux qui ont été faits dans cette optique, notamment par des gens comme Bierwisch, en Allemagne, sont fort importants. Seulement je crois que même dès le début des problèmes de morpho-syntaxe, de l'analyse de la morpho-syntaxe, on se heurte à des difficultés qui sont très grandes, parce que tout le reste de la chaîne les irrégularités de la norme, etc., reviennent vers le début, comme je pense le montrer par deux exemples que j'emprunte à l'exposé qui vient d'être fait. Dans un cas comme la série bûche, bûcher, bûcheron, bûcheronnage, je vois un certain nombre de processus qu'on peut étudier, qui ont une certaine forme de régularité, mais qui déjà sont hétérogènes et déjà posent un problème difficile. La relation entre bûcheron, bûcheronner, bûcheronnage est parfaitement régulière au point de vue morphosémantique. Les suffixes qui font passer de bûcheron, bûcheronnage à bûcheronner sont des suffixes de nominalisation entièrement productifs, entièrement vivants et entièrement actifs dans le système actuel du français. Par contre, de bûche à bûcheron, on a un passage qui n'est plus du tout régulier, parce que le suffixe n'est plus productif, parce que la régularité sémantique n'est plus totale et parce que je croix que, pour un Français moyen, la motivation est quasi nulle. Bûcheron a son statut, c'est un seul morphème et l'on peut 
l'opposer à bûche sans faire le passage de l'un à l'autre. C'est justement là une attitude intuitive qu'on peut choisir ou dans un sens ou dans l'autre, mais qui prouve bien qu'on ne peut pas se rapporter entièrement à la productivité et à la régularité du système. J'appellerai ce genre de morphème, comme le suffixe de bûcheron, des cadavres de morphèmes, parce qu'en fait ils existent à cause de raisons diachroniques mais ils ne sont plus productifs du tout, on ne peut plus rien en faire maintenant, ou on ne peut plus les utiliser productivement que dans des cas extrêmement spécifiques et particuliers. Donc déjà une première difficulté pour ce genre de chose dans lequel on a une hétérogénéité d'explication entre l'aspect diachronique où c'est parfaitement valable, et les aspects synchroniques où, à mon avis, cela ne l'est plus. D'autre part, on a l'apparition de ces deux morphèmes, vous avez tout à fait raison de souligner ce côté perpétuellement mouvant et productif des problèmes de lexique. Vous avez donné l'exemple de "gellule » qui est formé par le «ule» de capsule et «gel». On a naturellement là une formation qui est hybride au début, mais qui peut devenir productive. En français, ce n'est pas très actif, mais dans une langue comme l'anglais, il y a des exemples bien connus comme le suffixe burger qui a commencé avec hamburger et qui a donné cheeseburger, beefburger, etc. On a avec cette coupe arbitraire, qui est syllabique d'ailleurs et qui ne se base pas sur les morphèmes, des résultats absolument effarants et très actifs dans la langue publicitaire américaine. On a fait, par exemple, une publicité pour un produit préparé à base de riz qui s'appelait richghetti avec le pseudo suffixe "ghetti ", de "spaghetti " et qui maintenant marche dans un certain nombre de composés. On a ainsi un nouveau procédé morphologique qui à l'origine ne vient même pas d'un élément morphémique, mais d'un élément qui est purement une syllabe et qui n'a absolument aucun sens à priori. Cette mouvance de la productivité morphologique par disparition ou par apparition me pose le problème de l'applicabilité de règles totalement régulières. Ces règles doivent être étudiées; je ne critique pas du tout cet aspect de l'utilisation de la grammaire générative, mais je crois que la limite d'application est quand même plus étroite que votre exposé ne semblait le supposer. Je serais moins optimiste que vous pour les possibilités d'application de ces théories en lexicologie.

Réponse : C'est le premier point qui me paraît évidemment le plus difficile. Si on raisonne à partir de « bûche » et «bûcheron ", on a effectivement un suffixe qui n'est plus productif en français. Mais du point de vue de la grammaire générative, on peut faire abstraction de la productivité réelle ou non. Il suffit d'établir pour le locuteur la liaison entre "bûche » et "bûcheron " par une transformation quelconque. Le locuteur rétablit le rapport, la motivation. Même si théoriquement le suffixe n'est plus productif, il s'établit quand même une certaine motivation qui explique que "bûche * peut être en rapport avec « bûcheron ».

$M$. Rey : Je ne suis pas d'accord parce que le paradigme de "bûcheron" marche avec des mots comme arbre, comme forêt et pas du tout avec bûche. Le "bûcheron » n'est pas un monsieur qui fait des bûches, c'est un monsieur qui coupe les arbres. Par contre, je ne dis pas qu'il n'y ait pas toujours une possibilité, une tendance à la remotivation qui est d'ailleurs à la base du système de métaphoricité. C'est très important, mais au niveau connotatif. Je crois que le grand drame de l'applicabilité d'une linguistique théorique, que ce soit la grammaire générative ou une autre, à la lexicologie, c'est le saut direct de la compétence abstraite, ou du système abstrait, ou du schéma hjelmslevsien à la performance ou au discours. Ce ne sont pas des concepts identiques bien sûr, mais ils sont comparables.

Réponse : Nous ne sommes pas d'accord là-dessus, parce que je considère essentiellement le lexique du point de vue de la productivité. Je force peut-être un peu les rapports de motivation, mais il me semble que c'est l'essentiel, c'est-à-dire une théorie qui puisse rendre compte de cette création permanente qui a lieu dans le lexique même s'il y a des défaillances sur certains points.

$M$. Gauger : Je crois que nous avons là un très bon exemple de ce que j'appellerai une linguistique ouverte, c'est-à-dire une linguistique qui se concentre sur la chose, qui ne cherche pas à réaliser systématiquement des idées préconçues. Je crois qu'une interprétation syntagmatique des mots dérivés n'est pas possible, il faut voir là plutôt un type 
particulier de mots. Ce qui est intéressant dans cette discussion d'une manière plus générale évidemment, c'est que vous vous reportez ici à la conscience linguistique du locuteur moyen, c'est-à-dire à quelque chose de psychologique. Or on sait très bien que le structuralisme est ennemi de toute interprétation psychologique. Quelle différence faitesvous entre mot sémantique et mot référentiel ? Est-ce que vous pensez-là à deux types de mots différents ou à deux fonctions différentes du mot ou qu'ont la plupart des mots ? La plupart des mots sont à la fois mots sémantiques et mots référentiels ?

Réponse : L'appellation mot sémantique englobe les autres catégories, y compris mot référentiel et mot de discours. Je donne au mot sémantique le contenu le plus général. Donc " mot référentiel » n'est pas une catégorie par rapport au mot sémantique, mais un aspect de la signification du mot.
$M$. Baldinger : Il y a en effet une espèce de mode de production nouvelle. J'ai noté à peu près six ou sept exemples de formations à partir de « hôtel " : « motel » «botel » qui est un hôtel pour bébé, « rotel» un autobus où on peut coucher, une espèce d'hôtel roulant, "flotel », un hôtel en forme de bâteau, etc. Qu'est-ce qui reste du radical ? «b», " $\mathrm{m}$ ", etc., il n'y a plus de valeur propre et il est difficile de classer ces formations. Une remarque sur la génération par transformation des dérivés. Dans le tableau que j’ai distribué sur " garnir» et ses dérivés, il y a la relation horizontale, mais aussi la relation verticale, par exemple, "garnement " dans le sens de vaurien, ne se raccroche pas au verbe " garnir ", mais à un autre sens de "garnement *. Il faut donc toujours tenir compte des deux relations, des deux directions. Vous avez aussi parlé des trous dans la chaîne de création, mais il faut noter que certaines positions sont barrées, par exemple pour former le féminin de médecin. 